
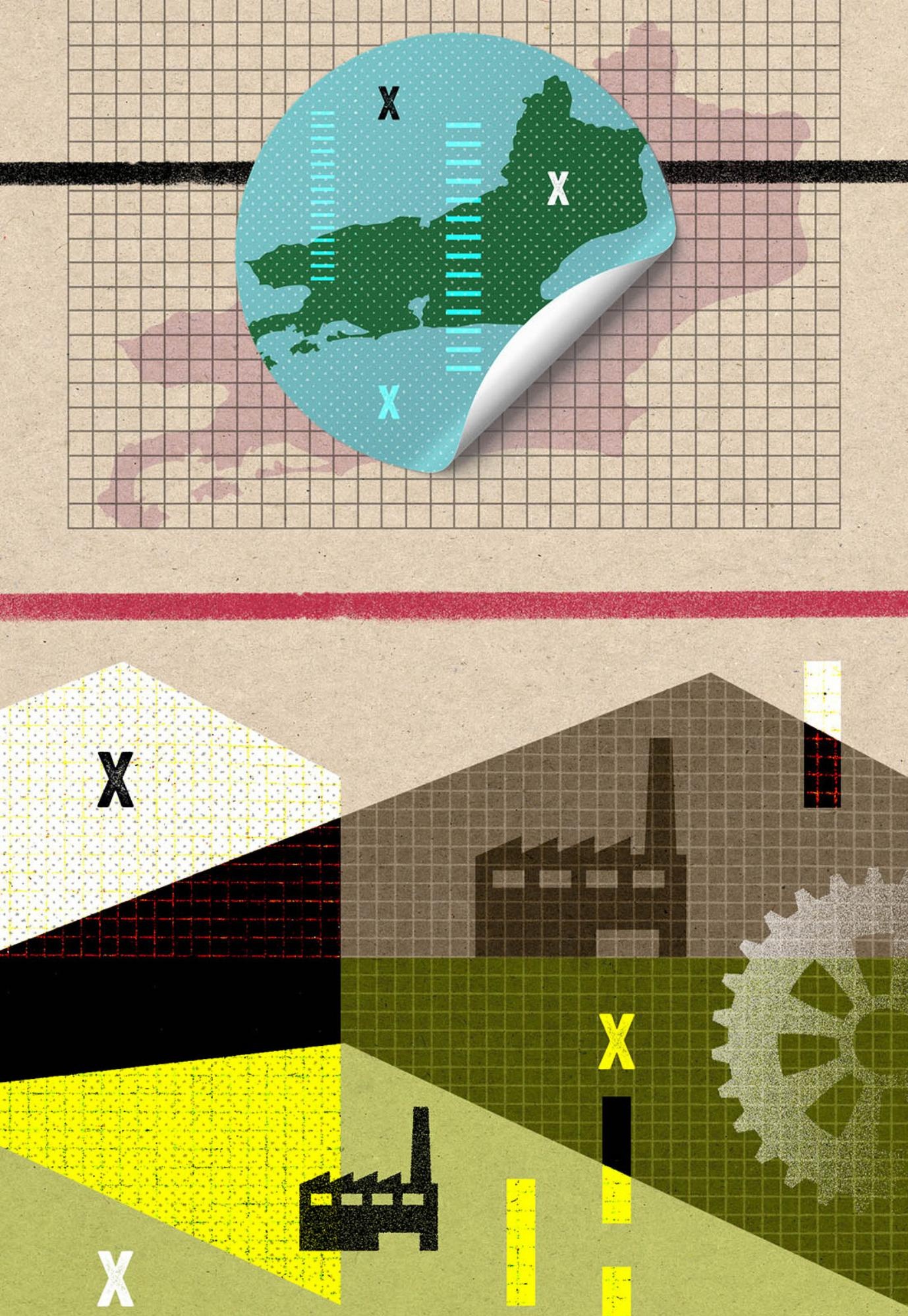


\title{
Exportações da indústria de transformação do estado do Rio de Janeiro e a hipótese de desindustrialização ${ }^{1}$
}

\author{
The transformation industry exports \\ in the state of Rio de Janeiro and the \\ deinsdustralization hypothesis
}

\author{
Daniel Arruda Coronel*| Leonardo Copetti** \\ http://dx.doi.org/10.51861/ded.dmvu.2.015 \\ Recebido em 16 de fevereiro de 2021. Aceito em 18 de junho de 2021.
}

\begin{abstract}
Resumo
Este trabalho tem como objetivo analisar o perfil industrial do Estado do Rio de Janeiro, visando responder à seguinte questão: está ocorrendo um processo de desindustrialização neste estado? Nesse sentido, foi ajustado um modelo Vetorial de Correção de Erro (VEC), com a variável dependente exportações da indústria de transformação do Estado do Rio de Janeiro. As variáveis independentes foram taxa de câmbio efetiva real; índice de preços das commodities; e coeficiente do grau de abertura comercial. Os resultados indicaram que a desvalorização cambial estimula as exportações, que a abertura comercial foi extremamente prejudicial às exportações e que a variável índice de preço das commodities apresentou sinal contrário ao esperado.
\end{abstract}

Palavras-chave: Indústria de Transformação. Taxa de Câmbio; Vetor de Correção de Erros.

\begin{abstract}
The present paper aims into analyze the industrial profile of the State of Rio de Janeiro, seeking to answer the following question: is a deindustrialization process taking place in this state? In this regard, a Vector Error Correction (VEC) was adjusted, with the dependent variable exports from the manufacturing industry in the State of Rio de Janeiro. The independent variable was the effective and real exchange rate; commodity price index; and coefficient of the commercial openness degree. The results indicated that the exchange rate devaluation stimulates exports, and that the trade opening was extremely detrimental to exports and the variable price index of commodities showed a sign contrary to expectations.
\end{abstract}

Keywords: Transformation Industry; Exchange Rate; Vector Error Correction.

\footnotetext{
* Daniel Arruda Coronel

Professor Associado do Departamento de Economia e Relações Internacionais, com atuação como Docente Permanente nos Programas de Pós-Graduação (Stricto sensu) em Gestão de Organizações Públicas, de Agronegócios e de Economia e Desenvolvimento, da Universidade Federal de Santa Maria (UFSM). Avenida Roraima, 1000. Cidade Universitária. Santa Maria, RS, Brasil. CEP: 97105-090.

ORCID: https://orcid.org/0000-0003-0264-6502.E-mail: daniel.coronel@uol.com.br

** Leonardo Copetti

Centro de Ciências Humanas, Universidade Federal de Santa Maria (UFSM). Avenida Roraima, 1000. Cidade Universitária. Santa Maria, RS, Brasil. CEP: 97105-090.

ORCID: https://orcid.org/0000-0002-4801-4714. E-mail: leonardocopetti@hotmail.com
} 


\section{INTRODUÇÃO}

O setor industrial é o motor do crescimento econômico, conforme Kaldor (1966); contudo, a indústria de transformação brasileira vem perdendo participação no Produto Interno Bruto (PIB) conforme dados do Instituto Brasileiro de Geografia e Estatística (IBGE, 2019), visto que sua participação, que chegou a perfazer $30 \%$ do PIB na década de 1980, passou para 13,3\% em 2012 e, caso continue nesse ritmo, a projeção para 2029 é de menos de $10 \%$.

Essa forte queda acendeu o debate de que a economia brasileira está passando por um processo de desindustrialização. Entre os que defendem a tese da desindustrialização, estão economistas como Cano (2012), Marconi (2015), Pochmann (2016), Bresser-Pereira (2009a; 2011; 2019) e Coronel (2019, 2020).

Contudo, existem vários economistas como Barros e Pereira (2008), Jank et al. (2008) e Bonelli e Pessoa (2010) que não compactuam desta visão, mas acreditam que o setor industrial brasileiro tem que superar desafios relacionados aos altos custos de transação, infraestrutura inadequada, problemas de logística, baixas taxas de investimento, elevada carga tributária, bem como instabilidade macroeconômica, o que tem feito com que a indústria venha perdendo competitividade.

Dada a importância do tema, vários trabalhos vêm sendo feitos visando analisar questões relacionadas à competitividade e à existência de um processo de desindustrialização na economia brasileira. Sonaglio et al. (2010) analisaram as evidências de um processo de desindustrialização na economia, por meio de dados em painel, e os resultados indicaram um possível processo de reprimarização da pauta de exportaçôes brasileiras, o qual pode ser evidenciado pela redução das exportações dos bens de alta intensidade tecnológica e aumento de bens não industriais.

Não obstante a isso, a maior parte dos estudos tem como foco o país, não abordando os impactos nas macrorregióes ou nos estados brasileiros, os quais têm peculiaridades que podem influenciar na dinâmica desse processo.

Nesse sentido, seguindo esta temática, mas com foco nos estados brasileiros, este trabalho tem como problema de pesquisa responder à seguinte questão: está ocorrendo um processo de desindustrialização no Estado brasileiro do Rio de Janeiro?

De acordo com o Ministério do Desenvolvimento, Indústria e Comércio (MDIC, 2020), o Estado do Rio de Janeiro foi responsável, em média, entre 2007 a 2019, por aproximadamente 10,35\% das exportaçóes totais do país, por $5,47 \%$ das exportaçôes da indústria de transformação, 33,17\% da indústria extrativa, $0,04 \%$ da agropecuária e $17,31 \%$ dos outros produtos. 
Apesar da importante participação, ainda há uma lacuna a ser preenchida visando analisar com maior acuidade o perfil exportador do estado, bem como para quais mercados estão direcionados os produtos da pauta exportadora e quais os produtos que mais se destacam.

A partir das respostas a essas questões, será possível formular estratégias e açôes visando aumentar a competitividade do estado, bem como políticas industriais que efetivamente sejam eficazes para uma reconfiguração industrial, não apenas meras reduções e isenções tarifarias.

Nesse sentido, o presente trabalho se propóe a mostrar empiricamente se esse estado passa por um processo de desindustrialização e quais variáveis econômicas podem auxiliar a reversão ou o aprofundamento desse processo, visando a uma reestruturação produtiva no setor industrial.

O presente trabalho está dividido em quatro seções, além desta introdução. A seção dois apresenta a revisão de literatura em que se explicita o conceito, as causas e as soluçóes para o processo de desindustrialização. Posteriormente, faz-se análise do perfil das exportaçóes do Estado do Rio de Janeiro. Na seção seguinte apresentam-se os procedimentos metodológicos utilizados na pesquisa. $\mathrm{Na}$ seção quatro, analisam-se os resultados da estimação do modelo e, por fim, são apresentadas as conclusões do trabalho, destacando-se as limitaçôes da pesquisa e as perspectivas para trabalhos futuros.

\section{REVISÃO DE LITERATURA}

\section{Desindustrialização: causas e consequências}

O crescimento econômico pode ser mais bem esboçado e analisado através das seguintes relações: a) existência de uma relação positiva entre o crescimento da indústria e o crescimento do produto agregado, ou seja, quanto maior for a taxa de crescimento do setor industrial, maior será a do produto nacional; b) uma correlação positiva entre o crescimento do produto industrial e da produtividade industrial; nesse sentido, constata-se uma relação de causalidade, pois, quanto maior for a taxa de crescimento da indústria, maior será a taxa de crescimento da produtividade; c) a longo prazo, o crescimento da economia não seria restrito pela oferta, mas pela sua demanda; nesse contexto, a restrição de demanda ao crescimento do produto em uma economia aberta seria o balanço de pagamentos (KALDOR, 1957; 1978).

Ainda nessa perspectiva, o setor industrial seria como um indutor do crescimento econômico, visto que este gera encadeamentos produtivos, economias de escala e externalidades para outros setores. Esse transbordamento das atividades industriais para os demais setores está relacionado à absorção 
de produtos e commodities produzidos no setor agrícola e de mineração, além da contratação de diversos tipos de serviços (KALDOR, 1966).

Para autores como Szirmai (2012), Acemoglu e Robinson (2012), Rodrik (2016), Bresser-Pereira, Oreiro e Marconi (2016) e Reinert (2016), todos os países que se desenvolveram tiveram seu modelo de crescimento puxado pelo setor industrial, visto que a produtividade nesse setor é maior do que na agricultura; o setor industrial oferece mais oportunidades de economias de escala que o setor agrícola, e os efeitos de encadeamento e transbordamento são maiores do que na agricultura.

A economia brasileira iniciou tardiamente o seu processo de industrialização, o qual teve um novo impulso a partir do Governo Vargas, na década de 1930, quando tem início o Processo de Substituição de Importaçóes (PSI), que vai até o final da década de 1980 e que adotou as seguintes medidas: desvalorização cambial; taxas múltiplas de câmbio; crédito e subsídios; elevadas tarifas de importação e forte participação do Estado, através de infraestrutura, logística e do arcabouço legal e institucional (CORONEL, AZEVEDO \& CAMPOS, 2014).

Da década de 1990 até meados de 2000, foram poucas as açóes para fomentar a competitividade do setor industrial, visto que o foco, no Brasil e demais países latino-americanos, era o controle das taxas de inflação e a busca pela estabilidade macroeconômica (PERES, 2006).

Ainda nessa perspectiva, a perda de competitividade do setor industrial brasileiro, ao longo dos anos 2000, começou a levantar debates e discussóes sobre se a economia brasileira estava passando por um processo de desindustrialização, o qual pode ser entendido como a redução persistente da participação do emprego industrial relativamente ao emprego total, bem como queda da participação do setor industrial no PIB (ROWTHORM \& RAMASWAMY, 1999; OREIRO \& FEIJO, 2010; BRESSER-PEREIRA, 2011, 2019).

De acordo com Bresser-Pereira (2011), os países desenvolvidos, a partir de certo nível de renda per capita, começam a se desindustrializar devido à concorrência de países em que a mão de obra é mais barata. Como consequência, esses países deixam de produzir bens industriais, especialmente de baixa tecnologia, transferindo sua mão de obra para setores de serviços com maior intensidade tecnológica e níveis de renda e de valor adicionado per capita mais alto, portanto, com salários médios mais altos. Quando esse processo ocorre desta forma, a desindustrialização não é prejudicial. No entanto, em países como o Brasil, que têm uma renda per capita baixa, esse processo de transformação estrutural é prematuro. 
Para Bresser-Pereira, Oreiro e Marconi (2016), quando um país passa por um processo de desindustrialização, algumas medidas devem ser adotadas, tais como desvalorização cambial, política industrial ativa, maiores investimentos em infraestrutura e sofisticação produtiva, ou seja, uma maior diversificação da produção com ênfase em setores de média e alta intensidade tecnológica.

Os principais efeitos do processo de desindustrialização em países em desenvolvimento como o Brasil são a) queda na renda dos trabalhadores; b) especialização regressiva, com retorno às vantagens comparativas baseadas em recursos naturais (Teoria das Vantagens Comparativas); c) tendência a desequilíbrios externos; d) quedas nas taxas de investimento; e) queda no Produto Interno Bruto (PIB); f) aumento da importação de produtos industriais, sendo que as causas para tal processo estão relacionadas sobrevalorização cambial, abertura econômica, altas taxas de juros, infraestrutura inadequada e alto custo Brasil (CANO; 2012, 2014; BENJAMIM, 2015).

Uma das formas de combater o processo de desindustrialização é por meio de políticas industriais, cujos principais instrumentos são a isenção tributária, a oferta de juros subsidiados, a discricionariedade da estrutura de tarifas de importação, a redução do Imposto sobre Produtos Industrializados (IPI) e do Imposto sobre Circulação de Mercadorias (ICMS) e medidas visando melhorar a infraestrutura e a redução de custos sistêmicos ou custos de transação (KUPFER, 2003; PACK \& SAGGI, 2006; CORONEL, AZEVEDO \& CAMPOS, 2014).

Desde 2000, três políticas industriais foram utilizadas para fomentar a competitividade do setor industrial brasileiro: a Política Industrial, Tecnológica e de Comércio Exterior (PITCE), que deixou como legado apenas a criação de marcos regulatórios, como a Lei da Inovação e da Biossegurança; a Política de Desenvolvimento Produtivo (PDP), que tinha como foco os setores de média-alta e alta intensidade tecnológica e se mostrou totalmente ineficiente para esses setores; por fim, a Política Brasil Maior, também com foco nos setores de alta intensidade tecnológica, mas que não atingiu boa parte de suas metas e ainda teve forte relação com a crise macroeconômica iniciada em 2014 (SOARES, CORONEL \& MARION FILHO, 2013; CORONEL, AZEVEDO \& CAMPOS, 2014; NASSIF, 2019; STUMM, NUNES \& PERISSINOTTO; 2019).

Padrão de especialização das exportações do Estado do Rio de Janeiro

Segundo o Instituto Brasileiro de Geografia e Estatísticas (IBGE, 2020), o Estado do Rio de Janeiro é o segundo estado brasileiro com maior participação no PIB, visto que, de 2007 a 2019, apresentou uma média de 11,74\%. 
De acordo com o Ministério do Desenvolvimento, Indústria e Comércio Exterior (MDIC, 2020), em 2019, as exportaçôes do Rio de Janeiro totalizaram US\$28,63 bilhões (2a posição no ranking de exportação dos estados brasileiros, com uma participação de $12,70 \%$ nas exportaçôes do país). No que tange às importações, o valor total foi de US\$21,21 bilhôes (2 ${ }^{a}$ posição nacional, com uma participação de 11,96\%).

Ainda segundo os dados do MDIC (2020), em 2019, a China foi o principal destino das exportaçóes do Estado do Rio de Janeiro, com um total de US\$13,58 bilhões, representando 47,43\% das exportações totais, seguida pelos Estados Unidos, com US\$ 6,04 bilhôes (21,08\%), e a Holanda, com US\$ 1,64 bilhóes $(5,73 \%)$. Os principais produtos exportados e suas participaçốes nas exportações totais foram petróleo com US\$19,31 bilhões (67,45\%), motores e máquinas não elétricos com US\$1,63 bilhões $(5,70 \%)$ e produtos semiacabados de ferro com US $\$ 1,52$ bilhôes $(5,31 \%)$.

Na Tabela 1, encontra-se a composição das exportaçóes do Estado do Rio de Janeiro por setor de atividade econômica, de 2007 a 2019.

Tabela 1 - Composição das exportações e importações, e as taxas geométricas de crescimento do Estado do Rio de Janeiro por setor da atividade econômica - 2007-2019

\begin{tabular}{|c|c|c|c|c|c|c|c|c|c|c|}
\hline \multicolumn{11}{|l|}{ Exportação } \\
\hline \multirow[t]{2}{*}{ Ano } & \multicolumn{2}{|c|}{ Agropecuária } & \multicolumn{2}{|c|}{$\begin{array}{l}\text { Indústria de } \\
\text { transformação }\end{array}$} & \multicolumn{2}{|c|}{$\begin{array}{l}\text { Indústria } \\
\text { extrativa }\end{array}$} & \multicolumn{2}{|c|}{$\begin{array}{l}\text { Outros } \\
\text { produtos }\end{array}$} & \multicolumn{2}{|c|}{ Total } \\
\hline & $\begin{array}{l}\text { Em } \\
\text { bilhões } \\
\text { de US\$ }\end{array}$ & $\%$ & $\begin{array}{l}\text { Em } \\
\text { bilhões } \\
\text { de US\$ }\end{array}$ & $\%$ & $\begin{array}{c}\text { Em } \\
\text { bilhões } \\
\text { de US\$ }\end{array}$ & $\%$ & $\begin{array}{l}\text { Em } \\
\text { bilhões } \\
\text { de US\$ }\end{array}$ & $\%$ & $\begin{array}{l}\text { Em } \\
\text { bilhões } \\
\text { de US\$ }\end{array}$ & $\%$ \\
\hline 2007 & 0,01 & 0,04 & 5,25 & 36,75 & 8,41 & 58,84 & 0,62 & 4,37 & 14,30 & 100,00 \\
\hline 2019 & 0,01 & 0,02 & 8,81 & 30,76 & 19,69 & 68,77 & 0,13 & 0,45 & 28,63 & 100,00 \\
\hline variação & 0,00 & $-0,02$ & 3,55 & $-6,00$ & 11,28 & 9,93 & $-0,49$ & $-3,92$ & - & - \\
\hline $\begin{array}{l}\text { taxas } \\
\text { geométricas de } \\
\text { crescimento }^{2}(\%)\end{array}$ & & 7,61 & & 5,99 & & 2,92 & & $-7,27$ & & 3,74 \\
\hline \multicolumn{11}{|l|}{ Importação } \\
\hline \multirow[t]{2}{*}{ Ano } & \multicolumn{2}{|c|}{ Agropecuária } & \multicolumn{2}{|c|}{$\begin{array}{l}\text { Indústria de } \\
\text { transformação }\end{array}$} & \multicolumn{2}{|c|}{$\begin{array}{l}\text { Indústria } \\
\text { extrativa }\end{array}$} & \multicolumn{2}{|c|}{$\begin{array}{c}\text { Outros } \\
\text { produtos }\end{array}$} & \multicolumn{2}{|c|}{ Total } \\
\hline & $\begin{array}{l}\text { Em } \\
\text { bilhões } \\
\text { de US\$ }\end{array}$ & $\%$ & $\begin{array}{l}\text { Em } \\
\text { bilhões } \\
\text { de US\$ }\end{array}$ & $\%$ & $\begin{array}{l}\text { Em } \\
\text { bilhões } \\
\text { de US\$ }\end{array}$ & $\%$ & $\begin{array}{l}\text { Em } \\
\text { bilhões } \\
\text { de US\$ }\end{array}$ & $\%$ & $\begin{array}{l}\text { Em } \\
\text { bilhões } \\
\text { de US\$ }\end{array}$ & $\%$ \\
\hline 2007 & 0,27 & 2,26 & 6,70 & 56,24 & 4,93 & 41,39 & 0,01 & 0,11 & 11,91 & 100,00 \\
\hline 2019 & 0,28 & 1,33 & 18,36 & 86,55 & 2,52 & 11,91 & 0,05 & 0,21 & 21,21 & 100,00 \\
\hline variação & 0,01 & $-0,94$ & 11,66 & 30,31 & $-2,40$ & $-29,48$ & 0,03 & 0,10 & - & - \\
\hline $\begin{array}{l}\text { taxas } \\
\text { geométricas de } \\
\text { crescimento (\%) }\end{array}$ & & $-0,82$ & & 5,21 & & $-9,12$ & & 12,55 & & 1,35 \\
\hline
\end{tabular}

Fonte: Elaboração dos autores a partir de dados da MDIC (2020) 
Pela análise da Tabela 1, observa-se uma redução de $6 \%$ na participação das exportações da indústria de transformação, passando de 36,75\%, em 2007, para $30,76 \%$ em 2019. O setor extrativo apresentou aumento de 9,93\%, os outros produtos apresentaram um declínio de 3,92 pontos percentuais e no setor agropecuário praticamente não houve alterações.

A análise da Tabela 1 demonstrou uma retração das importaçốes da agropecuária e da indústria extrativa, mas, por outro lado, aumento das importações da indústria de transformação. Tais resultados vão ao encontro de estudos como os Marconi (2015), Bender Filho e Coronel (2017), BresserPereira (2019) e também com o estudo feito pelo Banco Central do Brasil (BC, 2019) sobre penetração de importados e coeficiente de exportação da indústria de transformação, os quais apontaram que a sobrevalorização cambial do período foi um dos fatores que corroborou para o aumento das importaçóes de produtos de maior valor agregado e também contribuiu para o processo de desindustrialização.

Enfim, uma análise dos dados esboçados anteriormente, por mais simples que seja, mostra um aumento das exportaçóes da indústria extrativa do Estado do Rio de Janeiro, bem como decréscimo da participação de suas importaçóes e da agropecuária e um aumento na participação das importaçôes da indústria de transformação, o que tem forte relação com o câmbio apreciado e com a abertura econômica desordenada. A economia brasileira, a partir do Governo Collor, desde a década de 1990, não analisou detalhadamente as peculiaridades dos setores produtivos da economia brasileira, bem como as altas taxas de juros que inibem os investimentos internos e a infraestrutura inadequada, conforme autores como Coronel, Azevedo e Campos (2014), Peres et al. (2018) e Colombo, Felipe e Sampaio (2019).

\section{METODOLOGIA}

\section{Modelo Analítico}

O modelo Vetor Autorregressivo (VAR) é formado por um sistema de equaçôes que permite investigar os efeitos das variáveis endógenas nelas mesmas, bem como o impacto causado pelas mudanças em uma variável nas demais e a quantidade de variância em cada variável que pode ser atribuída às alteraçóes de cada variável nela mesma e nas demais (HAMILTON, 1994; BRANDT; WILLIAMS, 2007).

O modelo VAR, conforme Enders (2015), é expresso da seguinte forma:

$Y_{t}=\beta_{10}-\beta_{12} Z_{t}+Y_{11} y_{t-1}+Y_{12} Z_{t-1}+\varepsilon_{y t}$

$Z_{t}=\beta_{20}-\beta_{21} y_{t}+Y_{21} y_{t-1}+Y_{22} Z_{t-1}+\varepsilon_{z t}$

em que se assume que $Y_{t}$ e $Z_{t}$ são estacionários. 
Com base nisso, infere-se que as variáveis influenciam mutuamente umas às outras, tanto em relação ao tempo $t$ quanto à $t$-1. É por esse motivo que se utiliza o modelo VAR apenas quando há a confirmação de estacionariedade entre as séries, visto que, devido às variáveis contemporâneas $Z_{t}$ e $Y_{t}$ estarem individualmente correlacionadas com os erros $\varepsilon_{y t}$ e $\varepsilon_{z t}$, esse modelo não pode ser estimado diretamente, tendo em vista que cada variável depende contemporaneamente uma da outra (HARRIS, 1995; SHUMWAY \& STOFFER, 2006; LÜTKEPOHL, 2011).

Ainda nessa perspectiva, quando as variáveis forem não estacionárias, mas diferenciadas, estima-se um modelo de Vetor de Correção de Erros. Esse modelo torna-se necessário, pois permite a ligação entre os aspectos relacionados à dinâmica de curto e de longo prazo (HAMILTON, 1994; KOOP, 2006).

De acordo com Enders (2015) e Bueno (2015), em modelos de séries temporais, a primeira análise que deve ser feita é a determinação da ordem de integração das séries de interesse. Nesse sentido, utilizam-se os testes de raiz unitária de Dickey-Fuller Aumentado (DICKEY \& FULLER, 1979; 1981) e Kwiatkowski, Phillips, Schmidt e Shin (KPSS, 1992). Esses testes permitem verificar a existência ou não de raízes unitárias nas séries temporais, ou seja, se as variáveis são ou não estacionárias.

Um processo estocástico pode ser considerado estacionário quando a sua média e a sua variância forem constantes ao longo do tempo e quando o valor da covariância entre dois períodos de tempo depende apenas da distância, do intervalo ou da defasagem entre os períodos de tempo, e não do próprio tempo em que a covariância é calculada. Em notação matemática, as propriedades do processo estocástico estacionário podem ser representadas por: (Média) $E\left(Y_{t}\right)=\mu$, (Variância) $\operatorname{var}\left(Y_{t}\right)=E\left(Y_{t}-u\right)^{2}=\sigma^{2}$ e (Covariância) $\gamma_{k}=E\left[\left(Y_{t}-\mu\right)\left(T_{t+k}-\mu\right)\right]$. Um processo estocástico com as propriedades descritas anteriormente é conhecido, na literatura de séries temporais, como processo fracamente estacionário, ou estacionário em covariância, ou estacionário de segunda ordem, ou estacionário em sentido amplo. Um processo estocástico é fortemente estacionário quando todos os momentos de sua distribuição não variam ao longo do tempo (GREENE, 2008; BUENO, 2015.)

$\mathrm{O}$ teste $\mathrm{ADF}$ consiste na estimação da seguinte equação por Mínimos Quadrados Ordinários e pode ser expresso, conforme Enders (2015), da $\Delta y_{t}=\alpha_{0}+\gamma y_{t-1}+\sum_{i=2}^{p} \beta_{i} \Delta y_{t-i+1}+\varepsilon_{t}$

com: $\gamma=-\left(1-\sum_{i=1}^{p} \alpha_{i}\right)$ e $\beta_{i}=\sum_{j=i}^{p} \alpha_{j}$, em que: 
$\alpha_{0}$ é o intercepto;

$\gamma$ descreve o comportamento da série temporal;

$y$ representa a variável dependente;

$\Delta$ é a representação do operador de diferença; e

$\varepsilon_{t}$ denota o erro, que se assume ser idêntica e independentemente distribuída.

A fim de determinar o número de defasagens utilizadas no teste para eliminar a autocorrelação residual, utiliza-se o menor valor do critério de Schwarz (SBC) (ENDERS, 2015).

$\mathrm{O}$ parâmetro de interesse nas regressóes (sem intercepto e sem tendência; com intercepto; com intercepto e tendência) é $\gamma$, sendo que, se $\gamma=0$, a série contém uma raiz unitária. Nesse teste, compara-se o resultado da estatística $\tau$ com os valores apropriados reportados por Dickey-Fuller para determinar a aceitação ou a rejeição da hipótese nula $\gamma=0$. A hipótese nula será rejeitada se o valor calculado da estatística $\tau$ for maior do que o valor crítico de Dickey-Fuller, indicando que a série é estacionária; caso contrário, a série é não estacionária.

O KPSS é um teste alternativo ao ADF, sendo definido pelo procedimento de um teste de não estacionariedade contra a hipótese nula de estacionariedade, conforme Breitung, Brüggemann e Lütkepohl (2004) e Greene (2008), e pode ser formalmente expresso pela seguinte expressão:

$$
\begin{aligned}
& y_{t}=\alpha+\beta t+\gamma \sum_{i=1}^{t} z_{i}+\varepsilon_{t} \\
& y_{t}=\alpha+\beta_{t}+\gamma Z_{t}+\varepsilon_{t}
\end{aligned}
$$

tendo as seguintes hipóteses:

$H_{0}: \gamma=0$, série é estacionária;

$H_{a}: \gamma \neq 0$, série é não estacionária.

Mesmo que as variáveis individualmente não sejam estacionárias, mas exista pelo menos uma combinação linear estacionária entre elas, há a possibilidade de que essas variáveis sejam cointegradas (AMISANO \& GIANNINI, 1997; GREENE, 2008), ou seja, é possível verificar uma relação de equilíbrio de longo prazo entre elas, que pode ser estimada e analisada ${ }^{3}$. Dessa forma, para identificar o possível relacionamento de longo prazo entre as séries, utiliza-se o teste de cointegração elaborado por Johansen (1988). 
Antes de fazer o teste de cointegração, estima-se um VAR auxiliar, visando à determinação do processo de determinação do número de defasagens ótimas, também chamados de lags. Entre os critérios mais utilizados na literatura econômica de séries temporais, destaca-se o Valor da Verossimilhança (LogL); p-valor do teste de Razão de Verossimilhança (LR); Erro de Predição Final (FPE); Critério de Informação de Akaike (AIC); Critério de Informação Bayesiano de Schwarz (BIC); e o Critério de Informação de Hannan \& Quinn (HQC), conforme Lütkepohl (2007), Brooks (2008) e Bueno (2015).

O procedimento de Johansen $(1988,2014)$ considera que todas as variáveis são endógenas e sua utilização não é limitada pela existência de endogeneidade do regressor (relação causal no sentido da variável dependente para a variável explicativa). Esse procedimento utiliza Máxima Verossimilhança para estimar os vetores de cointegração e permite testar e estimar a presença de vários vetores de cointegração.

Conforme Johansen (2014), definido um vetor $z_{t}$ de $n$ variáveis potencialmente endógenas, é possível especificar o seguinte processo gerador, e modelar $z_{t}$ como um Vetor Autorregressivo (VAR) irrestrito com k defasagens de $z_{t}$ :

$$
Z_{t}=A_{1} Z_{t-1}+K+A_{k} Z_{t-k}+\varepsilon_{t}
$$

em que: $z_{t}$ é um vetor ( $\left.n x 1\right), A_{i}$ é uma matriz de parâmetros ( $\left.n x n\right)$ e $u_{t} \sim$ $\operatorname{IID}\left(\mu, \sigma^{2}\right)$.

Ainda conforme Johansen (2014), a Equação (5) pode ser reparametrizada em termos de um modelo Vetorial de Correção de Erro (VEC) esboçado como:

$$
\Delta z_{t}=\Gamma_{1} \Delta z_{t-1}+\ldots+\Gamma_{k-1} \Delta z_{t-k+1}+\prod z_{t-k}+\varepsilon_{t}
$$

em que: $\Gamma_{i}=-\left(I-A_{1}-\cdots-A_{i}\right),(\mathrm{i}=1, \ldots, \mathrm{k}-1)$ e $\Pi=-\left(I-A_{1}-\cdots A_{k}\right)$. O sistema contém informações de curto e longo prazo a mudanças de $z_{t}$, via estimativas de $\hat{\Gamma}_{i}$ e $\hat{\Pi}$, respectivamente, onde $\Pi=\alpha \beta^{\prime}$, com $\alpha$ representando a velocidade de ajustamento ao desequilíbrio e $\beta$ a matriz de coeficientes de longo prazo.

A importância do modelo de correção de erro reside no pressuposto de permitir a ligação entre aspectos relacionados à dinâmica de curto prazo com os de longo prazo (HAMILTON, 1994; LÜTKEPOHL 2007; 2011).

O número de vetores de cointegração depende do posto ou rank $(r)$ da matriz $\Pi$. Em termos de vetores de cointegração, tem-se três possibilidades, conforme Enders (2015): se o posto de é completo, então as variáveis $Y_{t}$ são 
I(0), ou seja, significa que qualquer combinação linear entre as variáveis é estacionária e o ajuste do modelo deve ser efetuado com as variáveis em nível; se o posto de $\Pi$ é zero, então não há relacionamento de cointegração e o modelo deve ser ajustado com as variáveis em diferença e, quando $\Pi$ tem posto reduzido, há $r$ vetores de cointegração (HAMILTON, 1994).

Ainda segundo o autor citado anteriormente, o rank de uma matriz é igual ao número de raízes características estritamente diferentes de zero, que pode ser identificado por meio de dois testes estatísticos. O primeiro deles é o teste do traço, que testa a hipótese nula de que o número de vetores de cointegração distintos é menor ou igual a $r$ contra a hipótese alternativa de que o número desses vetores é maior do que r, que pode ser definido por:

$$
\lambda_{\text {trace }}(r)=-T \sum_{i=r+1}^{n} \ln \left(1-\lambda_{i}^{\prime}\right)
$$

Em que

$\lambda_{i}^{\prime}=$ valores estimados das raízes características obtidos da matriz; $T=$ número de observações.

O segundo teste é o do máximo autovalor, que testa a hipótese nula de que o número de vetores de cointegração é $r$ contra a hipótese alternativa de existência de vetores de $r+1$ cointegração, podendo ser expresso da seguinte forma:

$$
\lambda_{\text {max }}(r, r+1)=-T \ln \left(1-\lambda_{r+1}^{\prime}\right)
$$

Verificada a cointegração entre as séries analisadas, estima-se o modelo VEC.

Posteriormente, fazem-se dois testes para verificar a presença de autocorrelação. Um deles é o teste de Portmanteau, o qual calcula as estatísticas BoxPierce / Ljung-Box multivariadas para correlação serial residual até a defasagem especificada.

Box-Pierce (1970) desenvolveram uma estatística Q baseada na soma dos quadrados das autocorrelaçóes. A estatística de Box-Pierce, $Q=T * \sum_{k=1}^{q} \rho_{k}^{2}$ comparada à distribuição Qui-quadrado com “q” graus de liberdade. Caso o resíduo seja um ruído branco, a estatística $Q$ definida segue uma distribuição Qui-quadrado com "q" graus de liberdade.

Ljung-Box (1978), conhecido como teste de Box-Pierce modificado, também é utilizado para verificar se há falha de ajuste em um modelo temporal. O teste examina as " $n$ ” primeiras autocorrelaçóes dos resíduos e, caso estas sejam pequenas, há indício de que não há falha no ajuste. A hipótese nula considera que as autocorrelações até o lag "n” são iguais a zero e a estatística do teste é dada por: 


$$
Q^{*}=T *(T-2) \sum_{k}^{n} \frac{\hat{\rho}_{k}^{2}}{T-k}
$$

onde $\rho \hat{k}$ é a autocorrelação estimada dos resíduos na defasagem " $\mathrm{k}$ ” e "n" o número de lags testado. O teste irá rejeitar a hipótese nula caso $Q^{*}>\chi_{1-\alpha ; q}^{2}$, sendo $\chi_{1-\alpha ; q}^{2}$ uma distribuição qui-quadrado com "q" graus de liberdade e nível de significância $\alpha$.

O teste de Breusch (1978) e Godfrey (1978), também conhecido como teste LM (Lagrange Multiplier) para autocorrelação, testa a hipótese nula de ausência de autocorrelação até a defasagem $p$. Após definir o modelo e estimar seus coeficientes, o teste consiste em efetuar uma regressão do resíduo contra o próprio resíduo defasado no tempo e as variáveis explicativas, de acordo com a Equação (10):

$$
\hat{u}_{t}=\alpha_{0}+\alpha_{1} x_{t}+\hat{\rho}_{1} \hat{u}_{t-1}+\hat{\rho}_{2} \hat{u}_{t-2}+\cdots+\hat{\rho}_{p} \hat{u}_{t-p}+v_{t}
$$

onde os últimos termos $\hat{u}_{t-1}, \hat{u}_{t-2}, \ldots, \hat{u}_{t-p}$ são os resíduos estimados pela equação do modelo adotado.

Visando responder ao problema de pesquisa e atingir os objetivos do presente trabalho, estimou-se um modelo econométrico com a indústria de transformação como variável dependente, baseados nos estudos de Veríssimo (2010), Veríssimo e Araújo (2016) e Souza e Veríssimo (2019).

As variáveis utilizadas para o ajuste do modelo foram:

INDTRANSF: exportações da indústria de transformação do Estado do Rio de Janeiro (RJ);

CAMBIO: taxa de câmbio efetiva real - exportações - Índice de Preços (IPA);

COM: índice de preços das commodities; e

ABERT: coeficiente do grau de abertura comercial - soma das exportaçóes e importaçôes totais em relação ao PIB do Estado do Rio de Janeiro (proxy para avaliar se a abertura comercial favoreceu a substituição da produção industrial interna por importaçôes).

Os dados para estimação dos parâmetros foram coletados nos seguintes sites: no Ministério do Desenvolvimento, Indústria e Comércio (MDIC, 2020), para as variáveis exportações do Estado do Rio de Janeiro, da indústria da transformação; no Instituto de Pesquisa Econômica Aplicada - IPEA (2019), para a variável taxa de câmbio - real efetiva - IPA - exportaçóes - (em reais por dólares - R\$/ US\$); no International Monetary Fund - IMF (2020), para a variável índice de preços das commodities; e no MDIC (2020) e no Instituto Brasileiro de Geografia e Estatística - IBGE (2020), para a variável coeficiente de abertura comercial do Estado do Rio de Janeiro (somatório das importaçóes 
e exportações totais, dividido pelo somatório do PIB dessa região). O período analisado foi o de fevereiro de 2007 a dezembro de 2019, com cotações mensais, totalizando 155 observaçóes.

Convém destacar que esse período compreende o início do II Governo Lula, quando as comodities tiveram um aumento expressivo em suas exportações - a crise do subprime -, e os Governos Dilma I, Dilma II e Temer, sendo que os dois últimos enfrentaram uma forte restrição macroeconômica, a qual afetou as exportaçôes brasileiras e contribuiu para um quadro de estagnação econômica.

Tabela 2 - Variáveis utilizadas no modelo e as respectivas siglas

\begin{tabular}{l|l}
\hline Variável & Sigla \\
\hline Exportações da indústria de transformação do Estado do Rio de Janeiro & INDTRANSF $^{4}$ \\
\hline Taxa de câmbio efetiva real - IPA & CAMBIO \\
\hline Índice de preços das commodities & COM \\
\hline Coeficiente de abertura comercial do Estado do Rio de Janeiro & ABERT \\
\hline Fonte: Organização dos autores &
\end{tabular}

$\mathrm{Na}$ Tabela 2, encontra-se um resumo das variáveis que foram utilizadas no modelo.

A série exportações da indústria da transformação do Estado do Rio de Janeiro (INDTRANSF) foi dessazonalizada pelo método Seazonal-trend Decomposition - STL do software Eviews 11. A seguir, foi aplicado o logaritmo neperiano sobre todas as séries e seus nomes foram alterados acrescentando um 'L' no início.

\section{ANÁLISE E DISCUSSÃO DOS RESULTADOS}

Testes de raiz unitária

Para dar início à estimação dos parâmetros dos modelos econométricos, foram realizados os testes de estacionariedade: ADF - Dickey-Fuller Aumentado; e KPSS - Kwiatkoswski, Philips, Schmidt e Shin. Os resultados estão expostos nas Tabelas 3 e 4 .

Com base na Tabela 3, observa-se que as séries das exportaçóes da indústria de transformação (LINDTRANSF) e coeficiente de abertura comercial (LABERT) foram estacionárias em nível, com $5 \%$ de significância para o teste $\mathrm{ADF}$, e a série índice de preços das commodities (LCOM) foi estacionária em nível, com 5\% de significância para o teste KPSS; já as demais variáveis não foram estacionárias em nível. Por outro lado, os resultados da Tabela 4 indicaram, em ambos os testes, efetuada a primeira diferença, que as séries foram estacionárias, com 5\% de significância. 
Tabela 3 - Testes de raiz unitária em nível aplicados nas séries mensais das exportações do Estado do Rio de Janeiro da indústria de transformação (US\$̦), taxa de câmbio efetiva real (R\$/ US\$), índice de preços das commodities e coeficiente de abertura comercial no período de fevereiro de 2007 a dezembro de 2019

\begin{tabular}{l|l|c|c|c|c|c}
\hline \multirow{2}{*}{ Séries } & \multicolumn{3}{|c|}{ Teste ADF } & \multicolumn{3}{c}{ Teste KPSS } \\
\cline { 2 - 7 } & Modelo & \multicolumn{2}{|c|}{ Estatística de teste } & Modelo & \multicolumn{2}{c}{ Estatística de teste } \\
\hline LINDTRANSF & constante & -5.446715 & $* * *$ & constante & 0.992521 & $* * *$ \\
\hline LCAMBIO & constante & -1.767729 & ns & constante & 0.819084 & $* * *$ \\
\hline LCOM & constante & -2.240245 & ns & constante & 0.462059 & $*$ \\
\hline LABERT & constante & -3.122869 & $* *$ & constante & 0.823113 & $* * *$ \\
\hline
\end{tabular}

Nota 1: *** significativo a 1\%; ** significativo a 5\%; * significativo a 10\%; ns não significativo Fonte: Elaboração própria com base nos dados da pesquisa

Tabela 4 - Testes de raiz unitária em primeira diferença aplicados nas séries mensais das exportações do Estado do Rio de Janeiro da indústria de transformação (US\$), taxa de câmbio efetiva real (R\$/ US\$), índice de preços das commodities e coeficiente de abertura comercial no período de fevereiro de 2007 a dezembro de 2019

\begin{tabular}{l|c|c|c|c|c|c}
\hline \multirow{2}{*}{ Séries } & \multicolumn{3}{|c|}{ Teste ADF } & \multicolumn{3}{c}{ Teste KPSS } \\
\cline { 2 - 6 } & Modelo & \multicolumn{2}{|c|}{ Estatística de teste } & Modelo & \multicolumn{2}{c}{ Estatística de teste } \\
\hline LINDTRANSF & constante & -12.46354 & $* * *$ & constante & 0.063284 & ns \\
\cline { 2 - 6 } LCAMBIO & constante & -9.506372 & $* * *$ & constante & 0.164818 & ns \\
\hline LCOM & constante & -7.285963 & $* * *$ & constante & 0.103813 & ns \\
\hline LABERT & constante & -13.61193 & $* * *$ & constante & 0.126634 & ns \\
\hline
\end{tabular}

Nota 1: *** significativo a $1 \%$; $^{* *}$ significativo a $5 \%{ }^{*}{ }^{*}$ significativo a $10 \%$; ns não significativo

Nota 2: Modelo com constante

Fonte: Elaboração própria com base nos dados da pesquisa

Efetuados os testes de estacionariedade e identificada a ordem de integração, a próxima etapa consistiu em analisar individualmente a dinâmica das exportações da indústria da transformação do Estado do Rio de Janeiro.

A fim de estimar os parâmetros do modelo econométrico que permitiu analisar as relaçôes entre a indústria de transformação (em US\$), com taxa de câmbio efetiva real (R \$/ US\$), índice de preços das commodities e coeficiente de abertura comercial, realizou-se o teste de cointegração de Johansen. Porém, para realizar esse teste, foi necessário, primeiramente, estimar um modelo VAR Auxiliar (Tabela 5). Para isso, foi essencial definir previamente o número ótimo de defasagens, por meio de um teste que permite identificar o comprimento dos lags.

Com base nos dados da Tabela 5, foi possível indicar que o número de defasagens é de três a serem incluídas no VAR, visto que, dos cinco critérios considerados, três indicaram o mesmo número de defasagens do VAR auxiliar.

Após determinados os números de defasagens do modelo, procedeu-se ao teste de cointegração de Johansen, que permite demonstrar a existência, ou 
não, de relações de longo prazo entre as variáveis. Os resultados do teste do autovalor e do teste do traço estão esboçados na Tabela 6.

O teste de cointegração de Johansen revelou que a hipótese nula de que não há nenhum vetor de cointegração deve ser rejeitada ao nível de 5\% para a estatística do traço e para a estatística do máximo autovalor. Além disso, o vetor de correção de erros que mais se ajustou foi o modelo 1 do sumário do teste, com as variáveis em nível sem tendência determinística e as equações de cointegração sem interceptos.

De acordo com a Tabela 7, que apresenta o vetor de cointegração (normalizado), todos os coeficientes estimados foram significativos ao nível de $1 \%$.

Tabela 5 - Critério de defasagem do VAR auxiliar para a indústria da transformação

\begin{tabular}{c|c|r|r|r|r|r}
\hline Defasagem & LogL & \multicolumn{1}{c|}{ LR } & FPE & AIC & SC & HQ \\
\hline 1 & 571.8506 & NA & $9.02 \mathrm{e}-09$ & -7.172266 & -6.858106 & -7.044661 \\
\hline 2 & 612.2847 & 76.69439 & $6.58 \mathrm{e}-09$ & -7.487545 & $-6.859225^{*}$ & $-7.232335^{*}$ \\
\hline 3 & 632.4580 & $37.22300^{*}$ & $6.24 \mathrm{e}-09^{*}$ & $-7.541394^{*}$ & -6.598914 & -7.158579 \\
\hline 4 & 639.9625 & 13.45966 & $6.98 \mathrm{e}-09$ & -7.431774 & -6.175134 & -6.921355 \\
\hline
\end{tabular}

Nota1:Valor da verossimilhança (LogL); $\mathrm{p}$-valor do teste de razão de verossimilhança (LR); Erro de Predição Final (FPE); Critério de Informação de Akaike (AIC); Critério de Informação Bayesiano de Schwarz (BIC); e 0 Critério de Informação de Hannan \& Quinn (HQC). Nota 2: *indica a defasagem ótima (n) definida pelo critério.

Fonte: Elaboração própria com base nos dados da pesquisa

Tabela 6 - Teste de cointegração de Johansen para a indústria da transformação

\begin{tabular}{l|l|l|l|l}
\cline { 2 - 5 } & \multicolumn{2}{c|}{$\begin{array}{c}\text { Estatística } \\
\text { do Traço }\end{array}$} & \multicolumn{2}{c}{$\begin{array}{c}\text { Estatística do Máximo } \\
\text { Autovalor }\end{array}$} \\
\cline { 2 - 5 } & Calculado & P-valor & Calculado & P-valor \\
\hline $\mathrm{R}^{5}=0$ & $45.36652^{* *}$ & 0.0138 & $30.94331^{* * *}$ & 0.0052 \\
\hline $\mathrm{R} \leq 1$ & 14.42321 & 0.5021 & 8.398597 & 0.6619 \\
\hline $\mathrm{R} \leq 2$ & 6.024608 & 0.4330 & 5.863216 & 0.3655 \\
\hline $\mathrm{R} \leq 3$ & 0.161392 & 0.7397 & 0.161392 & 0.7397 \\
\hline
\end{tabular}

Nota: *** significativo a $1 \%$; ** significativo a $5 \%$

Fonte: Elaboração própria com base nos dados da pesquisa

Tabela 7 - Estimativa do vetor de cointegração (normalizado) - indústria da transformação

\begin{tabular}{l|l|l|l}
\hline LINDTRANSF (-1) & LCAMBIO (-1) & \multicolumn{1}{|c|}{ LCOM (-1) } & LABERT $(-1)$ \\
\hline 1,0000 & $-1.339097^{* * *}$ & $-2.008506^{* * *}$ & $+1.450178^{* * *}$ \\
\hline & $(0.39113)$ & $(0.23248)$ & $(0.34944)$ \\
\hline & {$[-3.42367]$} & {$[-8.63933]$} & {$[4.14999]$} \\
\hline
\end{tabular}

Nota I: LINDTRANSF = logaritmo neperiano das exportações da indústria de transformação (US\$); LCAMBI0 = logaritmo neperiano da taxa de câmbio efetiva real (R\$/ US\$); LCOM = logaritmo neperiano do índice de preços das commodities; LABERT = logaritmo neperiano do coeficiente de abertura comercial. Nota 2: os valores entre parênteses referem-se ao erro padrão, e os entre colchetes, à estatística t. Nota $3:{ }^{* * *}$ significativo a $1 \% ;{ }^{* *}$ significativo a $5 \%$. 
$\mathrm{Na}$ Equação (11), é apresentado o vetor de cointegração (normalizado), indicando o relacionamento de longo prazo entre as variáveis com as respectivas elasticidades.

$$
\text { LINDTRANSF }_{t}=+1,34 \text { LCAMBIO }_{t}+2,01 \text { LCOM }_{t}-1,45 \text { LABERT }_{\mathrm{t}}
$$

De acordo com a Equação (11), verifica-se que um aumento de $1 \%$ na taxa de câmbio efetiva real (R\$/ US\$), ou seja, uma desvalorização cambial, ocasiona um aumento de 1,34\% nas exportações da indústria de transformação do Estado do Rio de Janeiro. Esse resultado vai ao encontro dos estudos de Bresser-Pereira (2012; 2019), Cano (2012), Veríssimo e Araújo (2016), Teixeira, Coronel e Oreiro (2019) e Copetti e Coronel (2020b), dentre outros economistas do Novo Desenvolvimentismo, que demonstraram empiricamente a importância da desvalorização cambial visando fomentar as exportações da indústria de transformação e estancar o processo de desindustrialização.

Ainda, de acordo com a Equação (11), o aumento de 1\% no índice de preços das commodities ocasiona um acréscimo de 2,01\% nas exportaçóes da indústria da transformação do Estado do Rio de Janeiro. Esse resultado também foi encontrado por Veríssimo e Araújo (2016), que analisaram o processo de desindustrialização no Estado de Minas Gerais, por Souza e Verissímo (2019), que analisaram o processo de desindustrialização na Região Sudeste do país e encontraram resultados positivos para os Estados de Minas Gerais e São Paulo. Conforme os autores, para Minas Gerais, uma possível explicação está relacionada às características de sua indústria baseada em recursos naturais e trabalho. Copetti e Coronel (2020a; 2020b), que analisaram o processo de desindustrialização dos Estados de Santa Catarina e Paraná, também corroboraram este resultado.

Também foi possível inferir, com base na Equação 11, que o aumento de $1 \%$ no coeficiente de abertura comercial ocasiona uma redução de $1,45 \%$ nas exportações da indústria da transformação do Estado do Rio de Janeiro. Esse resultado vai ao encontro dos estudos sobre desindustrialização de Palma (2005), Cano (2012), Bresser-Pereira (2019) e Copetti e Coronel(2020a; $2020 \mathrm{~b}$ ), dentre outros, que demonstraram a forma desordenada com que foi feita a abertura comercial no país.

Com a finalidade de diagnóstico do modelo, foram realizados dois testes de correlação serial para os resíduos, cujos resultados encontram-se na Tabela 8.

Com base na análise dos dados apresentados na Tabela 8, o teste, com até doze defasagens, indicou que não existem problemas de correlação serial, com $5 \%$ de significância. Dessa forma, os resultados de diagnóstico do modelo estimado são válidos e apresentam um relacionamento correto e confiável entre as variáveis em questão. 
Para a análise da dinâmica das variáveis do modelo, foi utilizado um instrumento proporcionado pelo modelo VAR/VEC, que é a decomposição da variância dos erros de previsão.

$\mathrm{Na}$ Tabela 9, apresentam-se os resultados relativos à decomposição da variância dos erros de previsão para as exportaçôes da indústria da transformação.

Os resultados da decomposição da variância dos erros de previsão das exportações da indústria da transformação (LINDTRANSF), apresentados na Tabela 9, mostram que, decorridos 24 meses após um choque não antecipado sobre essa variável, $63,60 \%$ de seu comportamento devem-se a ela própria, sendo que os $36,40 \%$ restantes são atribuídos às demais variáveis, $3,22 \%$ à taxa de câmbio efetiva real (LCAMBIO), 10,41\% ao índice de preços das commodities (LCOM) e $22,77 \%$ ao coeficiente de abertura comercial (LABERT).

Tabela 8 - Testes de correlação serial para os resíduos do modelo VEC

\begin{tabular}{c|c|l|l|l|l|l}
\cline { 2 - 7 } \multicolumn{5}{c|}{ Teste de Portmanteau } & \multicolumn{2}{c}{ Teste LM } \\
\hline Defasagens & Est. Q & p-valor & Est Ajust.Q & p-valor & Est. LM & p-valor \\
\hline 1 & 0.998841 & -- & 1.005327 & --- & 25.55608 & 0.0606 \\
\hline 2 & 4.046657 & --- & 4.092984 & --- & 20.83249 & 0.1850 \\
\hline 3 & 11.65417 & --- & 11.85064 & --- & 22.87200 & 0.1172 \\
\hline 4 & 26.38101 & 0.5521 & 26.96760 & 0.5200 & 25.58439 & 0.0602 \\
\hline 5 & 38.12124 & 0.7207 & 39.09917 & 0.6813 & 12.15922 & 0.7329 \\
\hline 6 & 61.13647 & 0.4349 & 63.04118 & 0.3693 & 24.27700 & 0.0836 \\
\hline 7 & 74.35325 & 0.5320 & 76.88309 & 0.4501 & 14.41744 & 0.5676 \\
\hline 8 & 88.65195 & 0.5795 & 91.95995 & 0.4816 & 15.19689 & 0.5103 \\
\hline 9 & 109.3891 & 0.4445 & 113.9754 & 0.3283 & 21.75480 & 0.1513 \\
\hline 10 & 117.2175 & 0.6540 & 122.3437 & 0.5252 & 8.323190 & 0.9387 \\
\hline 11 & 126.7464 & 0.7818 & 132.6005 & 0.6592 & 9.737105 & 0.8800 \\
\hline 12 & 133.5797 & 0.9028 & 140.0073 & 0.8160 & 7.458646 & 0.9634 \\
\hline
\end{tabular}

Fonte: Elaboração própria com base nos dados da pesquisa

Tabela 9 - Resultados da decomposição da variância dos erros de previsão em porcentagem para as variáveis LINDTRANSF, LCAMBIO, LCOM e LABERT no período de fevereiro de 2007 a dezembro de 2019

\begin{tabular}{l|c|c|c|c|c}
\hline Variável & Período (meses) & LINDTRANSF & LCAMBIO & LCOM & LABERT \\
\hline LINDTRANSF & 1 & 100.0000 & 0.000000 & 0.000000 & 0.000000 \\
\hline & 6 & 87.72940 & 1.362127 & 4.611033 & 6.297439 \\
\hline & 12 & 76.37689 & 2.147635 & 7.514042 & 13.96143 \\
\hline & 18 & 68.94272 & 2.771733 & 9.198073 & 19.08747 \\
\hline & 24 & 63.60146 & 3.220375 & 10.40668 & 22.77149 \\
\hline
\end{tabular}

Fonte: Elaboração própria com base nos dados da pesquisa 


\section{CONCLUSÕES}

O presente trabalho visava responder se está ocorrendo um processo de desindustrialização no Estado do Rio de Janeiro, e, neste sentido, utilizou-se de revisão bibliográfica, análise estatística e da estimação de um modelo econométrico VEC.

Os resultados indicaram que a participação das exportações do setor extrativo, de 2007 a 2019, aumentou, e a da indústria de transformação apresentou redução. Além disso, o Estado do Rio de Janeiro aumentou as importações da indústria de transformação e diminuiu as importaçôes da agropecuária e indústria extrativa, o que é um forte indício de uma mudança no seu perfil produtivo e pode caracterizar-se como um indício de desindustrialização.

Em relação à análise econométrica, constatou-se que a desvalorização cambial estimula as exportaçóes da indústria de transformação. A abertura comercial mostrou-se extremamente nociva às exportaçôes da indústria de transformação. A variável índice de preço das commodities apresentou sinal contrário ao esperado, contudo uma possível explicação para isso está relacionada ao perfil e à estrutura das exportaçóes do estado.

Com este trabalho, avançou-se no sentido de estudar o processo de desindustrialização para o Estado do Rio de Janeiro; contudo, não se pode generalizar os resultados para os demais estados brasileiros. Além disso, uma limitação do trabalho está relacionada às variáveis macroeconômicas utilizadas, ou seja, utilizou-se apenas três, as mais utilizadas na literatura econômica e nos trabalhos sobre desindustrialização. Contudo isto, de uma certa forma, limita um pouco a análise, visto que não contempla uma análise com maior acuidade sobre este processo.

Nesse sentido, sugere-se, para estudos futuros, a incorporação de outras variáveis macroeconômicas, bem como a estimação através de dados em painel com efeitos fixos e aleatórios, bem como a utilização de Modelos de Equilíbrio Geral Computável (MEGC), os quais são ferramentas que contribuem para verificar como o processo de desindustrialização influencia nos níveis de bem-estar da população, no Produto Interno Bruto (PIB), bem como nos fluxos econômicos e nas finanças públicas dos estados em análise. 


\begin{abstract}
Notas
' Este trabalho contou com aporte da FAPERGS e do CNPq, através do Edital Universal-2018.

2 Sequndo Greene (2008), a taxa geométrica de crescimento da variável Y pode ser descrita por: In $\mathrm{Y}_{\mathrm{t}}=\beta_{1}+\beta_{2} \mathrm{t}+\mathrm{u}_{\mathrm{i}}$, representando a função log-linear teórica para a taxa de crescimento "t", onde $\mathrm{u}_{\mathrm{i}}$ é um termo de erro aleatório, $\beta_{2}$ é a taxa de crescimento instantânea (em um ponto do tempo), e a taxa de crescimento composta (no decurso de um período) é calcula por $\left[\operatorname{anti} l o g\left(\hat{\beta}_{2}\right)-1\right] \times 100$

${ }^{3}$ Engle e Granger (1987) mostram que, se todas as séries de interesse possuem a mesma ordem de integração I(d) e existe um vetor $\alpha$, com $\alpha \neq 0$, em que a combinação linear dessas variáveis seja de or-

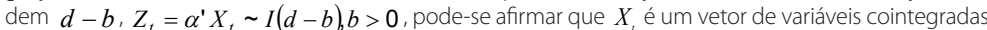
denotadas por $X_{t} \sim C I(d, b)$.

${ }^{4}$ Todas as séries foram transformadas em logaritmo natural com o intuito de normalizar os desvios.

${ }^{5}$ Indica o número de vetores de cointegração.
\end{abstract}

\section{Referências}

ACEMOGLU, D. \& ROBINSON, J. Por que as nações fracassam: as origens do poder, da prosperidade e da pobreza. Rio de Janeiro: Elsevier, 2012.

AMISANO, G. \& GIANNINI, C. Topics in structural VAR econometrics. 2. ed. Berlin: Spring-Verlag, 1997.

BANCO CENTRAL DO BRASIL (BC). Penetração de importados e coeficiente de exportação da indústria de transformação. 2019. Disponivel em: https://bit.ly/3rC8CPP. Acesso em: 21 de nov. 2019.

BARROS, O. De \& PEREIRA, R. R. Desmistificando a tese de desindustrialização: reestruturação da indústria brasileira em uma época de transformações globais. In: BARROS, O. de; GIAMBIAGI, F. (Org.). Brasil Globalizado. Rio de Janeiro: Elsevier, 2008.

BENDER FILHO, R. ACEMOGLU, D. \& ROBINSON, J. Por que as nações fracassam: as origens do poder, da prosperidade e da pobreza. Rio de Janeiro: Elsevier, 2012.

AMISANO, G \& GIANNINI, C. Topics in structural VAR econometrics. 2. ed. Berlin: Spring-Verlag, 1997.

BANCO CENTRAL DO BRASIL (BC). Penetração de importados e coeficiente de exportação da indústria de transformação. 2019. Disponivel em: https://bit.ly/3zFTy6y. Acesso em: 21 de nov. 2019.

BARROS, O. De \& PEREIRA, R. R. Desmistificando a tese de desindustrialização: reestruturação da indústria brasileira em uma época de transformações globais. In: BARROS, O. de; GIAMBIAGI, F. (Org.). Brasil Globalizado. Rio de Janeiro: Elsevier, 2008.

BENDER FILHO, R. \& CORONEL, D. A. Desindustrialização na economia gaúcha: evidências a partir de indicadores de orientação externa. In: CALANDRO, M. L.; MIEBACH, A. D.; ALVIM, A. M. (Org.). Inovação, sustentabilidade e desenvolvimento no RS. Porto Alegre: FEE, 2017.

BENJAMIN, C. Desindustrialização: pode o Brasil sobreviver sem um expressivo setor industrial? Boletim Conjuntura Brasil, Fundação João Mangabeira, n. 2, out. 2015.

BONELLI, R. \& PESSOA, S. Desindustrialização no Brasil: um resumo da evidência. Centro de Desenvolvimento Econômico, FGV, 2010. (Texto para Discussão). Disponível em: https://bit.ly/373DjUv. Acesso em: 04 maio 2017.

BONELLI, R.; PESSOA, S. \& MATOS, S. Desindustrialização no Brasil: fatos e interpretação. In: BACHA, E. \& BOLLE, M. B. (Org.). O futuro da indústria no Brasil: desindustrialização em debate. Rio de Janeiro: Civilização Brasileira, 2013.

BOX, G. E. P. \& PIERCE, D. A. Distribution of residual autocorrelations in autoregressive integrated moving average time series models. Journal of the American Statistical Association, v. 65, n. 332, pp. 1509-1526, 1970. 
BRANDT, P. T. \& WILLIAMS, J. T. Multiple Time Series Models. California: Sage, 2007.

BREITUNG, J; BRÜGGEMANN, R. \& LÜT$\mathrm{KEPOHL}, \mathrm{H}$. Structural vector autoregressive modeling and impulse responses. In: LÜTKEPOHL, H. \& KRÄTZIG, M. (Ed.). Applied time series econometrics. New York: Cambridge University Press, 2004.

BROOKS, C. Introductory Econometrics for Finance. New York: Cambridge University Press, 2008.

BRESSER-PEREIRA, L. C. Getúlio Vargas: o estadista, a nação e a democracia. 2009a Disponível em: https://bit.ly/3yaZrrZ. Acesso em: 15 fev. 2019.

BRESSER-PEREIRA， L. C. Globalização $e$ competição: porque alguns países emergentes têm sucesso e outros não. Rio de Janeiro: Elsevier, 200gb.

BRESSER-PEREIRA, L. C. A taxa de câmbio no centro da teoria do desenvolvimento. 2011. Disponível em: https://bit.ly/3rEERoX. Acesso em: 3 jun. 2019.

BRESSER-PEREIRA, L. C. Desprotecionismo e desindustrialização. 2012. Disponível em:

https://bit.ly/37juuX3. Acesso em: 10 de out. 2019.

BRESSER-PEREIRA, L. C. 40 anos de desindustrialização. 2019. Disponível em: https:// bit.ly/3ygemDh. Acesso em: 03 nov. 2019.

BRESSER-PEREIRA，L C; OREIRO, J L. \& MARCONI, N. Macroeconomia desenvolvimentista. Rio de Janeiro: Campus. 2016.

BREUSCH, T. S. Testing for autocorrelation in dynamic linear models. Australian Economic Papers, v. 17, 1978.

BUENO, R. L. S. Econometria de séries temporais. 2. ed. São Paulo: Cengage Learning, 2015.

CANO, W. A desindustrialização no Brasil. Economia e Sociedade, v. 21, Número Especial, pp. 831-851, 2012.

CANO, W. (Des)Industrialização e (Sub)Desenvolvimento. Cadernos do desenvolvimento, v. 9, n. 15, pp. 139-174, 2014.
COLOMBO, A. O.; FELIPE, E. S. \& SAMPAIO, D. A desindustrialização no Brasil: um processo, várias vertentes. In: ENCONTRO INTERNACIONAL DA ASSOCIAÇÃO KEYNESIANA BRASILEIRA. Anais... Campinas (SP) IE-UNICAMP, 2019.

COPETTI, L. S. \& CORONEL, D. A. Perfil industrial das exportações do estado de santa catarina e a hipótese de desindustrialização. Revista Eletrônica do Alto Vale do Itajaí - REAVI, v. 9, n. 15, pp. 96-119, 2020 a.

COPETTI, L. S. \& CORONEL, D. A . Perfil industrial das exportações do estado do Paraná e a hipótese de desindustrialização. In: XVIII ENABER - Encontro Nacional da Associação Brasileira de Estudos Regionais e Urbanos, 2020, Encontro Virtual. Anais do XVIII ENABER - Encontro Nacional da Associação Brasileira de Estudos Regionais e Urbanos. v. 1. pp. 1-20, 2020 b.

CORONEL, D. A. Impactos da política de desenvolvimento produtivo na economia brasileira. Curitiba: Prismas, 2013.

CORONEL, D. A. Processo de desindustrialização da Economia Brasileira e possibilidades de reversão. Revista de Economia e Agronegócio, v. 17, n.3 pp. 389-398, 2019.

CORONEL, D. A. Análise do processo de desindustrialização na Região Sudeste do Brasil: uma abordagem por meio de econometria de séries temporais. Monografia (Especialização em Estatística e Modelagem Quantitativa) - Universidade Federal de Santa Maria, 2020.

CORONEL, D. A.; AZEVEDO, A. F. Z. De \& CAMPOS, A. C. Política industrial e desenvolvimento econômico: a reatualização de um debate histórico. Revista de Economia Política, v. 34, n. 1, pp. 103-119, 2014.

DICKEY, D. A. \& FULLER, W. A. Distribution of the estimators for autoregressive time series with a unit root. Journal of the American Statistical Association, v. 74, n. 366, pp. 427-431, 1979. 
DICKEY, D. A. \& FULLER, W. A Likelihood ratio statistics for autoregressive time series with a unit root. Econometrica, v. 49, n. 4, pp. 1057-1073، 1981.

ENDERS, W. Applied Econometric Time Series. 4. ed. Nova Jersey: Wiley, 2015.

ENGLE, R. F. \& GRANGER, C. W. Co-integration and error-correction: representation, estimation and testing. Econometrica, Chicago, v. 55, n. 2, pp. 251-276, 1987.

FERREIRA, P. C. \& HAMDAN, G. Política industrial no Brasil: ineficaz e regressiva. Econômica, Rio de Janeiro, v. 5, n. 2, pp. 305316, 2003.

FUNDAÇÃO CENTRO DE ESTUDOS DO COMÉRCIO EXTERIOR (FUNCEX). Estatísticas. Disponível em: https://bit.ly/2WmXFGg. Acesso em: 18 ago. 2019.

GELATTI, E. et al. Desindustrialização no brasil: uma análise à luz das exportações e importações - 1997 a 2018. In: SALÃO DO CONHECIMENTO - BIOECONOMIA: DIVERSIDADE E RIQUEZA PARA O DESENVOLVIMENTO SUSTENTÁVEL - UNIJUÍ - XXIV Jornada de Pesquisa, 2019، Panambi-RS-Brasil. Anais... Panambi-, 2019.

GODFREY, L. G. Testing against general autoregressive and moving average error models when the regressor include lagged dependent variables. Econometrica, v. 46, n. 6, pp. 1293-1302, 1978.

GREENE, W. H. Econometrics Analysis. 6. ed. New Jersey: Pearson Education, 2008.

HAMILTON, J. D. Time series analysis. New Jersey: Princeton University Press, 1994.

HARRIS, R.I.D. Using cointegration analysis in econometric modelling. London: Prentice Hall-Harvester Wheatsheaf, 1995.

INSTITUTO DE PESQUISA ECONÔMICA APLICADA. IPEA. Macroeconômico. Disponível em: https://bit.ly/2ViuuU7. Acesso em: 14 jul. 2019.

INTERNATIONAL MONETARY FUND IMF. Primary commodity price system. Disponível em: https://bit.ly/3i6nwKY. Acesso em: $\mathrm{O} 2$ jun. $202 \mathrm{O}$.
JANK, M. S. et al. Exportações: existe uma "doença brasileira"? In: BARROS, O. de; GIAMBIAGI, F. (Org.). Brasil Globalizado. Rio de Janeiro: Elsevier, 2008.

JOHANSEN, S. Statistical analysis of cointegration vectors. Journal of Economic Dynamic and Control, v. 12, n. 2-3, pp. 231-254, 1988.

JOHANSEN, S. Times series cointegration. Creates Research Paper, n. 38, 2014.

KALDOR, N. A model of economic growth. Economic Journal, v. 67, n. 268, pp. 591-624, 1957.

KALDOR, N. Causes of the slow rate of economic growth of the United Kingdom. Cambridge: Cambridge University Press, 1966.

KALDOR, N. Causes of the low rate of growth of the United Kingdom. Further Essays in Economic, Growth, London Duckworth, 1978.

KOOP. G. Analysis of financial data. New Jersey: John Wiley \& Sons Ltd, 2006.

KWIAKOWSKI, D. et al. Testing the alternative of stationary against the alternative of a unit root: how sure are we that economic time series have a unit root? Journal of Econometrics, v. 54, p. 159-178, 1992.

KUPFER, D. Política industrial. Econômica, Rio de Janeiro, v. 5, n. 2, p. 281-298, 2003.

LIBÂNIO, G.; MORO, S. \& LONDE, A. C. Qualidade das exportações e crescimento econômico nos anos 2000. ENCONTRO NACIONAL DE ECONOMIA-ANPEC, 42. Anais... Natal, 2014.

LJUNG, G. M. \& BOX, G. E. P. On a measure of lack of fit in time series models. Biometrika, v. 65, n. 2 pp. 297-303, 1978.

LÜTKEPOHL, H. New introduction to multiple time series analysis. New York: Springer, 2007.

LÜTKEPOHL, H. Vector autoregressive models. EUI Working Paper, n.30, 2011.

MARCONI, N. A doença holandesa e o valor da taxa de câmbio. In: OREIRO, J. L.; DE PAULA, L. F. de. \& MARCONI, N. (Org.). A teoria econômica na obra de Bresser-Pereira. Santa Maria: Ed. UFSM, 2015. 
MATTEI, L. \& SCARAMUZZI, T. A taxa de câmbio como instrumento do desenvolvimento econômico. Revista de Economia Política, v. 36, n. 4, p. 726-747, 2016.

MINISTÉRIO DO DESENVOLIMENTO INDÚSTRIA E COMERCIO EXTERIOR (MIDIC). Secretaria de Comércio Exterior (SECEX). Disponivel em: https://bit.ly/3loyoof. Acesso em: 31 dez. 2018.

NASSIF. A. Política industrial e desenvolvimento econômico: teoria e propostas para o Brasil na era da economia digital. In: FEIJO, C. \& ARAÚJO, E. (Orgs.). Macroeconomia moderna: lições de Keynes para economias em desenvolvimento. Rio de Janeiro: Elsevier, 2019.

OREIRO, J. L. \& FEIJÓ, C. A. Desindustrialização: conceituação, causas, efeitos e o caso brasileiro. Revista de Economia Política, v. 30, n. 2, p. 219-232, 2010.

ORGANIZAÇÃO PARA A COOPERAÇÃO E DESENVOLVIMENTO ECONÔMICO OCDE. Industry and entrepreneurship. Disponível em: https://bit.ly/3rECafD. Acesso em: 28 out. 2019.

PALMA, J. G. Four sources of deindustrialization and a new concept of the Dutch disease. In: OCAMPO, J. A. (Ed.). Beyond Reforms. Palo Alto: Stanford University Press, 2005.

PACK, H. \& SAGGI, $K$. Is there a case for industrial policy? A critical survey. The Word Bank Research Observer, v. 21, n. 2, p. 267-297, 2006.

PEREIRA, W. M. \& CARIO, S. A. F. Indústria, desenvolvimento econômico e desindustrialização: sistematizando o debate no Brasil. Economia e Desenvolvimento, v. 29, n.1, p. 587-609, 2017.

PERES, W. The slow comeback of industrial policy in Latin America and the Caribbean. CEPAL Review, Chile, v. 88, n. 1, p. 71-88, 2006.

PERES, S.C. et al. Uma investigação sobre os determinantes da desindustrialização: teorias e evidências para países desenvolvidos e em desenvolvimento (1970-2015). In:
ENCONTRO DE ECONOMIA DA REGIÃO SUDESTE (ANPEC-SUL), 21., 2018, Curitiba-PR-Brasil. Anais... 2018.

PINHEIRO, M. C. et al. Porque o Brasil não precisa de política industrial. Rio de Janeiro: FGV, 2007. (Texto para discussão 644).

PINTO, N. G. M. ; SILVA, M. L. \& CORONEL, D.A. O processo de desindustrialização: um levantamento sobre a produção científica na base do Scopus. Revista de Administração da UEG, v. 6, n. 3, pp. 78-88, 2015.

POCHMANN, M. Brasil sem industrialização: a herança renunciada. Ponta Grossa: Editora da UEPG, 2016.

REINERT, E. S. Como os países ricos ficaram ricos... e porque os países pobres continuam pobres. Rio de Janeiro: Contraponto, 2016.

RODRIK, D. Premature deindustrialization. Journal of Economic Growth, v. 21, pp. 1-33, 2016.

ROWTHORN, R. \& RAMASWAMY, R. Growth, trade and deindustrialization. Washington: International Monetary Fund Staff Papers, v. 46, n. 1, 1999.

SHUMWAY, R. H. \& STOFFER, D. S. Times series analysis and its applications with $R$ examples. New York: Springer, 2006.

SILVA, J.A. A desindustrialização na Região Sudeste. Cadernos Metropolitanos, São Paulo, v. 21, n. 45, p. 531-550, 2019.

SILVA, M. L. et al. O setor industrial brasileiro frente à integração econômica. Belo Horizonte: Poisson, 2019.

SOARES, C. S.; CORONEL, D. A. \& MARION FILHO, P. J. A recente política industrial brasileira: da política de desenvolvimento Produtivo ao Plano Brasil Maior. Perspectivas Contemporâneas, v. 8, n. 1, pp. 1-20, 2013. SONAGLIO, C. M. et al. Evidências de desindustrialização no Brasil: uma análise com dados em painel. Economia Aplicada, v. 14, n.4, pp. 347-372, 2010. 
SOUZA, I. E. \& L. De \& VERÍSSIMO, M. P. Produção e emprego industrial nos estados brasileiros: evidências de desindustrialização. Nova Economia (UFMG), v. 29, n. 1, pp. 75-101, 2019.

SQUEFF, G. C. Desindustrialização: luzes e sombras no debate brasileiro. Brasília: IPEA, 2012. (Texto para discussão 1747).

STRACK. D. \& AZEVEDO, A. F. Z. A doença holandesa no Brasil: sintomas e efeitos. Economia e Desenvolvimento, v. 24, n. 2, pp. 68-91, 2012.

STUMM, M. G.; NUNES, W. \& PERISSINOTTO, R. Ideias, instituições e coalizões: as razões do fracasso da política industrial lulista. Revista de Economia Política, v. 39, n. 4, pp. 736-754, 2019

SZIRMAI, A. Industrialisation as an engine of growth in developing countries, 19502005. Structural change and economic $d Y$ namics, v. 23, n. 4, pp. 406-420, 2012.

TEIXEIRA, F. O. et al. Grau de especialização da cesta de exportações e sua relação com o crescimento econômico dos estados brasileiros. In: ENCONTRO DE ECONOMIA DA
REGIÃO SUDESTE (ANPEC-SUL), 21., 2018, Curitiba-PR-Brasil. Anais, 2018.

TEIXEIRA; F. O. CORONEL, D. A. \& OREIRO, J. L. principais determinantes do comportamento e da intensidade tecnológica das exportações brasileiras. In: ENCONTRO INTERNACIONAL DA ASSOCIAÇÃO KEYNESIANA BRASILEIRA. Anais. Campinas (SP) IE-UNICAMP, 2019.

VERÍSSIMO, M. P. Doença holandesa no Brasil: Ensaios sobre taxa de câmbio, perfil exportador, desindustrialização e crescimento econômico. 2010. Tese (Doutorado em Economia) - Universidade Federal de Uberlândia, 2010.

VERÍSSIMO, M. P. \& ARAÚJO, S. C. Perfil Industrial de Minas Gerais e a hipótese de desindustrialização estadual. Revista Brasileira de Inovação. Campinas, v. 15, n.1, pp. 113-138, jan./jun. 2016.

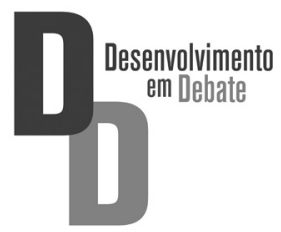

